\title{
Dryophthorinae (Coleoptera: Curculionidae) in the Collection of the Museu Paraense Emílio Goeldi: inventory and type specimens Dryophthorinae (Coleoptera: Curculionidae) da Coleção do Museu Paraense Emílio Goeldi: inventário e espécimes-tipo
}

\author{
Roberta de Melo Valente (10 | José Raimundo Rocha Guimarães' (1) \\ Universidade Federal do Pará. Instituto de Ciências Biológicas. Laboratório de Invertebrados. Belém, Pará, Brasil
}

\begin{abstract}
This paper reports the results of the curation of specimens of Dryophthorinae (Coleoptera: Curculionidae) housed in the Entomological Collection of the Museu Paraense Emilio Goeldi (MPEG), Belém, state of Pará, Brazil. The Collection holds a total of 1,071 specimens, with $90 \%$ of them previously unidentified. We reviewed all specimens and identified them to species level, resulting in 16 genera and 32 species of Dryophthorinae. These include the type specimens of three species: Cyrtomasius marluciae Vanin, 1998; Foveolus maculatus O'Brien, 2003; and Paramasius cristulatus Vanin, 1998. The genus Rhodobaenus Le Conte, 1876 was recorded for the first time to Brazilian Amazon. All specimen data were digitized and are now publicly available. Currently, $50 \%$ of the richness of the Dryophthorinae recorded from Brazilian Amazon is present in the Collection. The occurrence and the species richness of the Dryophthorinae examined are shown on a map. All labels contents and remarks to type material are presented. High resolution photographs of the primary types are also provided. The material examined represents a temporal and spatial record of Amazonian Dryophthorinae.
\end{abstract}

Keywords: Weevil. Richness. Taxonomy. Cyrtomasius. Paramasius. Foveolus.

Resumo: Este artigo reporta os resultados da curadoria dos espécimes de Dryophthorinae (Coleoptera: Curculionidae) depositados na Coleção Entomológica do Museu Paraense Emílio Goeldi (MPEG), Belém, Pará, Brasil. O acervo soma 1.071 exemplares, sendo que $90 \%$ deles não tinham sido identificados anteriormente. Nós revisamos todos os espécimes e os identificamos em nível de espécie, resultando em 16 gêneros e 32 espécies de Dryophthorinae. Estes incluem os espécimes-tipo de três espécies: Cyrtomasius marluciae Vanin, 1998; Foveolus maculatus O'Brien, 2003; e Paramasius cristulatus Vanin, 1998. O gênero Rhodobaenus Le Conte, 1876 foi registrado pela primeira vez na Amazônia brasileira. Todas as informações presentes nas etiquetas do material estudado foram digitadas e agora estão disponíveis para consulta. Atualmente, 50\% da riqueza de espécies de Dryophthorinae registrada na Amazônia brasileira está representada na coleção. A ocorrência e a riqueza de espécies de Dryophthorinae registradas na coleção são apresentadas em um mapa. Todas as informações das etiquetas do material estudado e os comentários sobre o material-tipo são fornecidos. Fotografias de alta resolução dos tipos estudados também são fornecidas. O material examinado representa um registro temporal e espacial de Dryophthorinae da Amazônia.

Palavras-chave: Gorgulho. Riqueza. Taxonomia. Cyrtomasius. Paramasius. Foveolus.

VALENTE, R. M. \& J. R. R. GUIMARÃES, 2021. Dryophthorinae (Coleoptera: Curculionidae) in the Collection of the Museu Paraense Emílio Goeldi: inventory and type specimens. Boletim do Museu Paraense Emílio Goeldi. Ciências Naturais 16(1): 33-50. DOI: http:// doi.org/10.46357/bcnaturais.v16i1.306.

Autora para correspondência: Roberta de Melo Valente. Universidade Federal do Pará. Instituto de Ciências Biológicas. Laboratório de Invertebrados. Rua Augusto Corrêa, 01 - Guamá. Belém, PA, Brasil. CEP 66075-110 (roberta.mvalente@gmail.com).

Recebido em 13/05/2020

Aprovado em 05/09/2020

Responsabilidade editorial: Valéria Juliete Silva
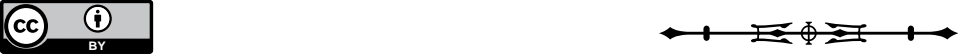


\section{INTRODUCTION}

Curculionidae Latreille, 1802 is one of the most diverse groups of insects, with about 4,600 genera and 51,000 described species in the world (Oberprieler et al., 2014). Even though one of the most recognizable families of Coleoptera, the classification of the subfamilies and tribes of Curculionidae remains highly controversial (about classification within Curculionidae, e.g. Kuschel, 1995; Marvaldi \& Morrone, 2000; Marvaldi et al., 2002; McKenna et al., 2009, 2018; Shin et al., 2018). As an exception to the rule, Dryophthorinae Schoenherr, 1825 is one of the few well-defined groups within Curculionidae, supported both by molecular and morphological data (Kuschel, 1995 as Rhynchophorinae; Marvaldi, 1997 as Rhynchophorinae; Marvaldi \& Morrone, 2000; Anderson, 2002a; Marvaldiet al., 2002; Oberprieler et al., 2007; Anderson \& Marvaldi, 2014; McKenna et al., 2009; Jordal et al., 2014; Shin et al., 2018).

Dryophthorinae (the subfamilies's content is equal to that of "Dryophthoridae" by Alonso-Zarazaga \& Lyal, 1999, 2002a; and Bouchard et al., 2011) consists about 1,200 species in 153 genera and five tribes: Cryptodermatini, Dryophthorini, Stromboscerini, Orthognathini (with two subtribes) and Rhynchophorini (with six subtribes) (Marvaldi \& Morrone, 2000; Oberprieler et al., 2007; Morrone \& Cuevas, 2009). Their species are mainly associated with monocots (Anderson, 2002a; Marvaldi et al., 2002; McKenna et al., 2009), and some species are major pests of various globally important agricultural products (Silva et al., 1968; Chamorro, 2019).

In South America are recorded 27 genera of Dryophthorinae (Wibmer \& O'Brien, 1986; Vanin, 1998; Alonso-Zarazaga \& Lyal, 1999, 2002a), which of 17 were taxonomically studied. Thereby, all the richest genera (Metamasius Horn, 1873; Mesocordylus Lacordaire, 1866; Sphenophorus Schoenherr, 1838; and Rhodobaenus Le Conte, 1876) were reviewed by Vaurie (1966, 1967b, 1970b, 1978, 1980) including Cactophagus Le Conte, 1876 and Paramasius Kuschel, 1958 (as species group of Metamasius; see Vaurie, 1966, 1967a). Other four genera with less richness
(Belopoeus Schoenherr, 1838; Rhinostomus Rafinesque, 1815; Orthognathus Schoenherr, 1838; and Scyphophorus Schoenherr, 1838) were also reviewed by Vaurie (1968c, 1970a, 1970b, 1971). Additionally, she described Foveolus Vaurie, 1968 and furnished an identification key to its species (Vaurie, 1968b). Other important studies were the review of Rhynchophorus Herbst, 1795 and Dynamis Chevrolat, 1883 (Wattanapongsiri, 1966) and, more recently, the description of the genus Cyrtomasius Vanin, 1998 (Vanin, 1998) besides the review of Melchus Lacordaire, 1866 (Anderson, 2003), Eucalandra Faust, 1899 (Anderson, 2008a) and Alloscolytroproctus Hustache, 1929 (Anderson, 2008b). The remaining of the genera of Dryophthorinae from South America is represented by one to four species in the region, moreover some of them can be identified by a species key (e.g. Kuschel, 1961 to species of Sitophilus Schoenherr, 1838) or by an identification key present in the taxonomic reviews cited above.

The Entomological Collection of the Museu Paraense Emílio Goeldi (MPEG), located in Belém, Pará, Brazil, represents the second oldest entomological depository in Brazil (Costa et al., 2000). This centenary Collection includes more than 11,000 mounted non-type specimens of Curculionidae, of which about $50 \%$ have been identified to genus or species level, summing 216 genera and 149 species (Costa et al., 2000; Valente et al., 2005). Additional information on the Collection is available at MPEG (n. d.). The Dryophthorinae are represented by type specimens of three species, and $90 \%$ of the remaining specimens housed in the Entomological Collection at MPEG remained until now unidentified.

Herein, we summarize the diversity of Dryophthorinae housed in the Entomological Collection at MPEG, including the identification to the species level for all of the specimens. Following a recommendation of the International Code of Zoological Nomenclature (ICZN, 1999, p. 72F.4), we also include type specimens of Dryophthorinae deposited in this Collection, providing photographs and relevant taxonomic notes. 


\section{MATERIAL AND METHODS}

We examined type and non-type specimens of Dryophthorinae housed in the Entomological Collection of the Museu Paraense Emílio Goeldi. Identification relied on published genus and species-group level reviews (Anderson, 2002b; Vaurie, 1966, 1967a, 1967b, 1968a, 1968b, 1968c, 1970a, 1970b, 1977, 1978, 1980; Wattanapongsiri, 1966), original descriptions and comparison with previously identified material from this Collection. Type specimen's current status was verified by the following databases: Catalogue of Life (Roskov et al., 2020); Electronic Catalogue of Weevil Names of Curculionoidea (Alonso-Zarazaga \& Lyal, 2002b); and Taxonomic Catalog of the Brazilian Fauna (Vanin et al., 2020). Species are ordered by tribes, subtribes and genera in alphabetical order, according to the classification proposed by Alonso-Zarazaga \& Lyal (1999) with modifications by Marvaldi \& Morrone (2000). The names of genera and species are followed by author(s) and year of publication.

The following data are provided for type specimens: bibliographic references, number of total specimens examined, category of types, sex when it is indicated, code-number of MPEG, and all labels contents in verbatim. Backslash indicates a line break, square brackets enclose the contents of a single label and double quotes enclose all the labels for a given specimen. In the remarks, there is additional information on specimens. For non-type specimens the following data are provided: the total number of specimens examined, country, state, city, locality (when indicated), date, biological information (when indicated), collectors (when indicated) and number of specimens examined. Additional information (not originally present in the labels) are enclosed in square brackets. The repeated information was removed and indicated by "same data". The section "Occurrence (MPEG Collection)" presents only the contents from the database provided in this study. All specimens examined received a code-MPEG COL follow by a number of eight digits, according to the present rules of the MPEG Entomological Collection. Label contents were added to the Collection database available at GBIF and MPEG (n. d.).
The map of occurrence and species richness of Dryophthorinae deposited in the MPEG Entomological Collection was generated from the database provided in this study. Data occurrence not found or, limited to the name of the country or state were not recorded on the map, which were indicated by asterisk (*) in the material examined.

Photographs of the holotypes and paratypes were made using a digital camera DFC420 attached to a Leica MZ16 magnifying stereoscope. Leica LAS (Leica Application Suite V4.6.1) and Helicon Focus (version 6.0.18) software were used to assemble the series of images taken at different focus distances by z-stacking method.

\section{RESULTS AND DISCUSSION}

A total of 1,071 Dryophthorinae specimens were examined belonging to 16 genera and 32 species (Table 1), including type specimens of three species: Cyrtomasius marluciae Vanin, 1998 (Paratype, Figure 1), Foveolus maculatus O'Brien, 2003 (Paratype, Figure 2), and Paramasius cristulatus Vanin, 1998 (Holotype, Figure 3). In this study, 934 specimens were identified and represented an increase of 13 species to Dryophthorinae richness in the Entomological Collection at MPEG. Two specimens of Sphenophorus were identified as two morphotypes and seven specimens of Metamasius remain unidentified at species level. The remaining 128 specimens were previously identified in 19 species by the specialists D. R. Whitehead, S. A. Vanin and C. W. O'Brien. All material examined is listed below.

In Brazil, Dryophthorinae is represented by species classified in the Orthognathini and Rhynchophorini tribes, summing 90 species in 22 genera (see Wibmer \& O'Brien, 1986; Vanin, 1995, 1998; Alonso-Zarazaga \& Lyal, 1999; O'Brien, 2003). Among them, 61 species in 20 genera (except Eucalandra and Rhodobaenus) have been recorded from Brazilian Amazon (Table 1). Therefore, the Entomological Collection at MPEG includes 50\% of the species richness of the Dryophthorinae recorded from Brazilian Amazon. Considering only this fauna, five genera are fully represented also five genera are absent in the Collection (Table 1). 
The genus Rhodobaenus was recorded for the first time to Brazilian Amazon while the genus Sphenophorus was recorded for the first time in the MPEG Entomological Collection. In the Brazilian Amazon are known five species as endemic to the region (Table 1): Belopoeus orbignyae Bondar, 1954; Belopoeus heikeae Vanin, 1995; C. marluciae; F. maculatus and; P. cristulatus (Wibmer \& O'Brien, 1986; Vanin, 1995, 1998; O'Brien, 2003), of which only B. orbignyae and $B$. heikeae are absent in the Collection. The richest genera in the Collection were Metamasius and Mesocordylus (both with six species), and the most abundant species were Metamasius hemipterus (Linnaeus, 1758) (390 specimens)

Table 1. Number of species of Dryophthorinae known for the Brazilian Amazon, known as endemic to Brazilian Amazon, and number of species represented in the MPEG Entomological Collection. Notation: $(-)=$ none; $(*)=$ morphotypes not included in the total.

\begin{tabular}{|c|c|c|c|}
\hline Genus & Brazilian Amazon & Known as endemic & MPEG collection \\
\hline \multicolumn{4}{|l|}{$\begin{array}{l}\text { Orthognathini } \\
\text { Orthognathina }\end{array}$} \\
\hline Mesocordylus Lacordaire, 1866 & 6 & - & 6 \\
\hline Orthognathus Schoenherr, 1838 & 3 & - & 2 \\
\hline \multicolumn{4}{|l|}{ Rhinostomina } \\
\hline Rhinostomus Rafinesque, 1815 & 2 & - & 1 \\
\hline \multicolumn{4}{|l|}{$\begin{array}{l}\text { Rhynchophorini } \\
\text { Rhynchophorina }\end{array}$} \\
\hline Dynamis Chevrolat, 1883 & 3 & - & 3 \\
\hline Rhynchodynamis Heller, 1906 & 2 & - & - \\
\hline Rhynchophorus Herbst, 1795 & 2 & - & 1 \\
\hline \multicolumn{4}{|l|}{ Litosomina } \\
\hline Neophrynoides O'Brien \& Wibmer, 1982 & 1 & - & - \\
\hline Sitophilus Schoenherr, 1838 & 3 & - & 1 \\
\hline Toxorhinus Lacordaire, 1866 & 1 & - & 1 \\
\hline \multicolumn{4}{|l|}{ Sphenophorina } \\
\hline Alloscolytroproctus Hustache, 1929 & 1 & - & - \\
\hline Belopoeus Schoenherr, 1838 & 5 & 2 & 2 \\
\hline Cactophagus Le Conte, 1876 & 10 & - & 2 \\
\hline Cosmopolites Chevrolat, 1885 & 1 & - & 1 \\
\hline Cyrtomasius Vanin, 1998 & 1 & 1 & 1 \\
\hline Foveolus Vaurie, 1968 & 4 & 1 & 2 \\
\hline Metamasius Horn, 1873 & 9 & - & 6 \\
\hline Paradiaphorus Chevrolat, 1885 & 1 & - & - \\
\hline Paramasius Kushel, 1958 & 3 & 1 & 2 \\
\hline Rhodobaenus Le Conte, 1876 & - & - & 1 \\
\hline Scyphophorus Schoenherr, 1838 & 1 & - & - \\
\hline Sphenophorus Schoenherr, 1838 & 2 & - & $2 *$ \\
\hline TOTAL & 61 & 5 & 32 \\
\hline
\end{tabular}

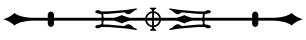




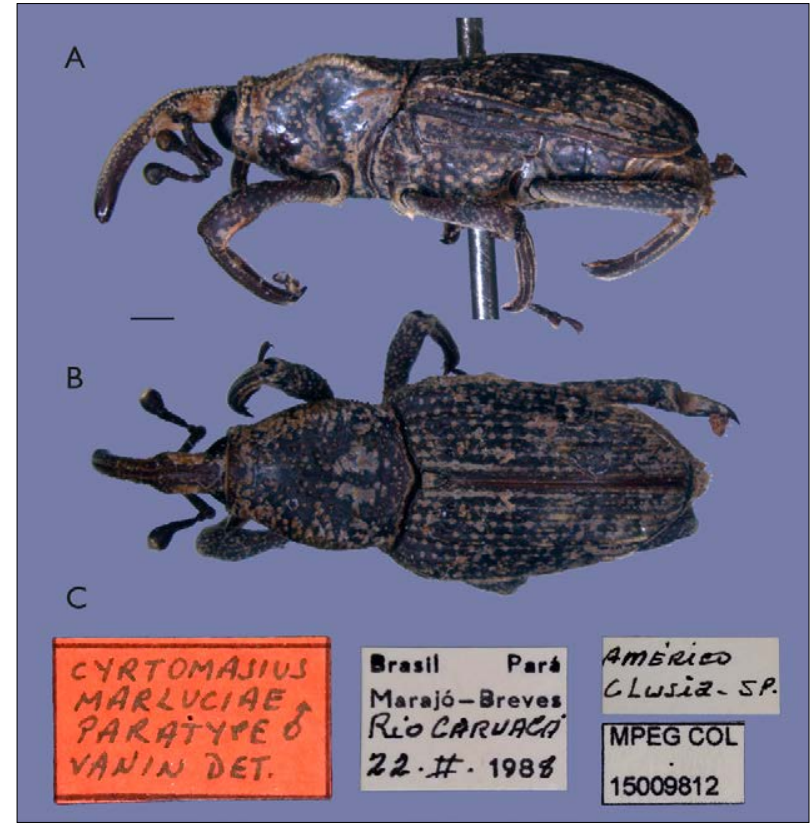

Figure 1. Paratype male of Cyrtomasius marluciae Vanin, 1998: A) lateral view; B) dorsal view; C) labels. Scale $=1 \mathrm{~mm}$.

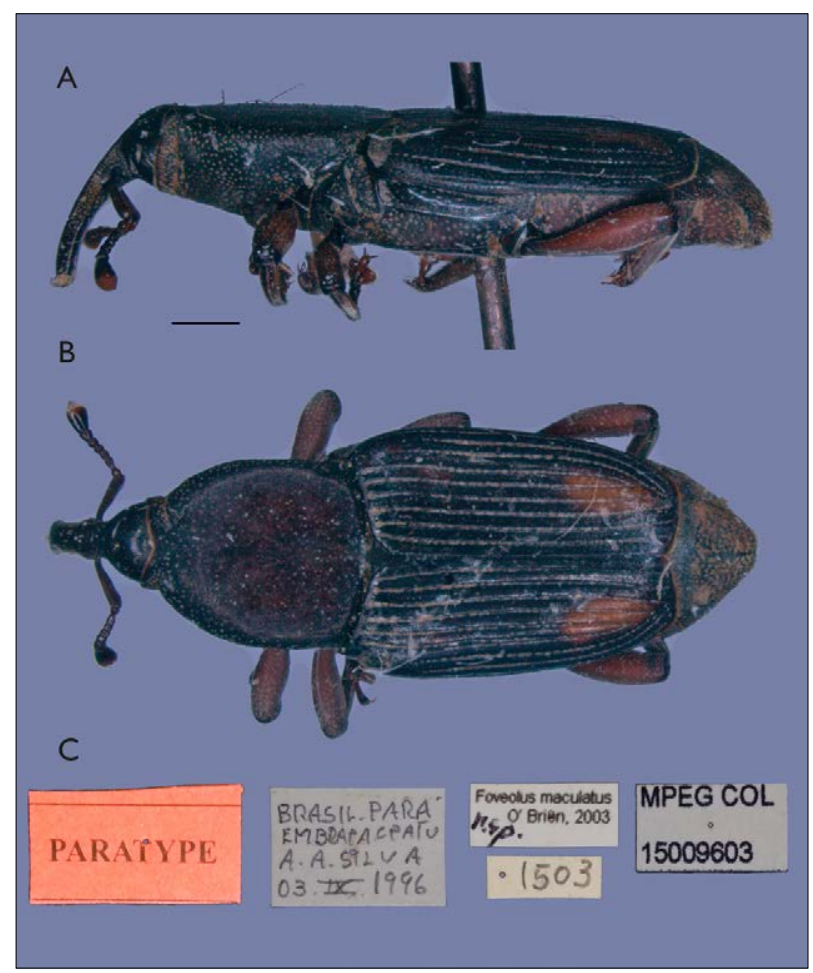

Figure 2. Paratype female of Foveolus maculatus O'Brien, 2003: A) lateral view; B) dorsal view; C) labels. Scale $=1 \mathrm{~mm}$.

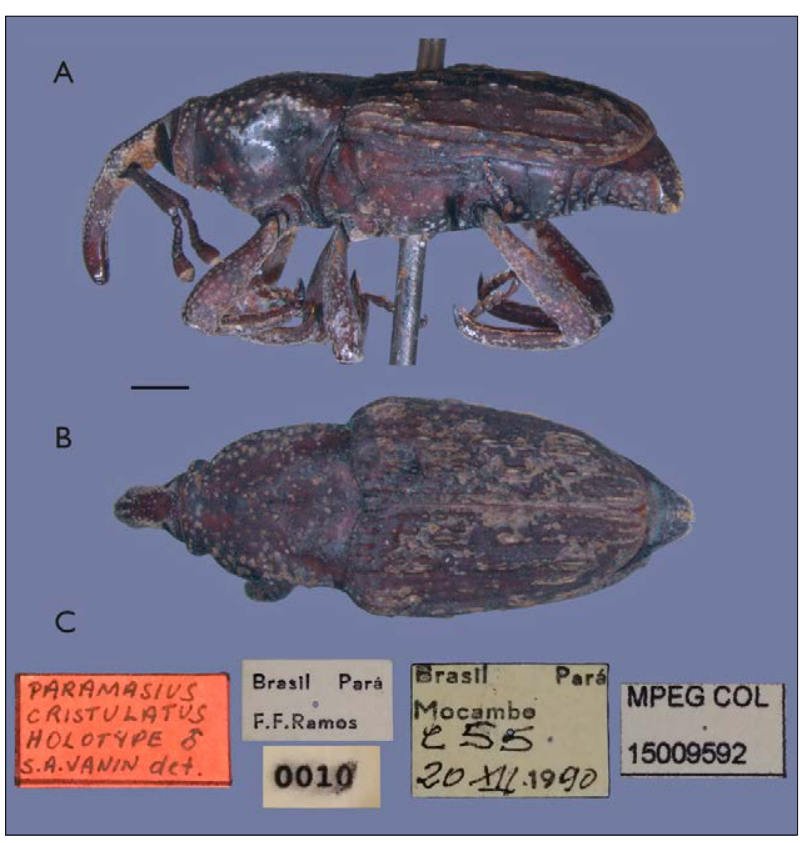

Figure 3. Holotype male of Paramasius cristulatus Vanin, 1998: A) lateral view; B) dorsal view; C) labels. Scale $=1 \mathrm{~mm}$.

and Rhynchophorus palmarum (Linnaeus, 1758) (389 specimens). In contrast, five species were represented by a single specimen each (see the list below).

Many of specimens examined were collected casually, so little biological or ecological information have been recorded on them. Exceptions are cited to specimens of Dynamis borassi (Fabricius, 1801); Rhynchophorus palmarum; Belopoeus carmelita Gyllenhal, 1838; Belopoeus caudatus; Foveolus anomalus Vaurie, 1968; Foveolus maculatus and Metamasius hemipterus on several palms and; Cyrtomasius marluciae and Paramasius cristulatus on Clusia (see the list below).

The material examined was collected exclusively in Brazil (from 90 municipalities from 13 states), especially from the state of Pará (from 40 municipalities) (Figure 4). The highest value of the species richness of Dryophthorinae in the MPEG Entomological Collection was also recorded from the state of Pará (29 species), followed by the states of Amazonas and Mato Grosso (both with eight species) (Figure 4). These occurrences do not necessarily represent the geographic distribution of the species, they are mainly

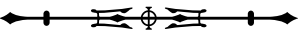




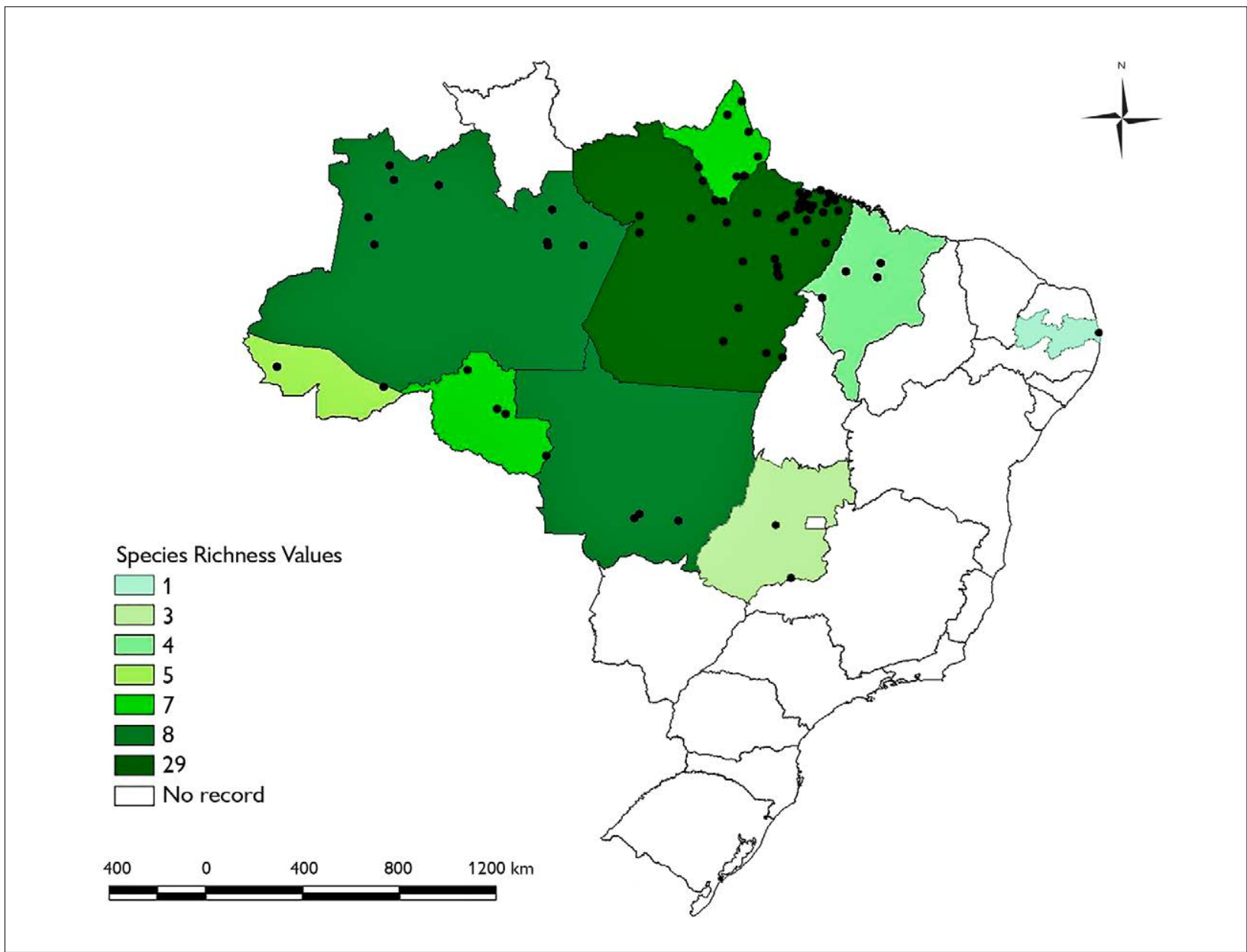

Figure 4. Occurrence and species richness of the Dryophthorinae deposited in the MPEG Entomological Collection. Points indicate the localities recorded in the material examined.

the result of surveys carried out over the years by the staff of MPEG. Nevertheless, the material examined also includes specimens from regions already affected by human intervention, especially from Belém, Tucuruí, and Parauapebas, all in the state of Pará. Besides specimens collected by Adolpho Ducke in the early 1900s' and, Walter Egler, Jan Karel Bechyné, and Bohumila Springlová Bechyné in the early 1960's. Thereafter, a significant amount was added to the Collection as result of the surveys conducted by the staff of MPEG in the 1970-1990's, specially Drs. Bento Melo Mascarenhas, William Leislie Overal, and Inocêncio de Sousa Gorayeb, together the assistants: Ramiro Bittencourt
Neto, Waldemar Miranda da Silva França, Francisco Ferreira Ramos, Maria Fernanda Pinto Torres, Márcio Zanuto, and José Orlando Dias.

\section{LIST OF SPECIES OF DRYOPHTHORINAE IN THE MPEG ENTOMOLOGICAL COLLECTION}
Curculionidae
Dryophthorinae
Orthognathini
Orthognathina

Mesocordylus Lacordaire, 1866

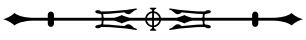


Mesocordylus apiciclava Vaurie, 1970

Material examined: 1 specimen. Brazil: Rondônia: Ji-Paraná, Fazenda Sinueira, 29-IX-1989, M. Zanuto Coll. (1).

Occurrence (MPEG Collection): Brazil: Rondônia (ji-Paraná).

\section{Mesocordylus cerinus Vaurie, 1970}

Material examined: 2 specimens. Brazil: Pará: Parauapebas, Caldeirão, Serra Norte, 27-X-1984, M.F. Torres Coll. (2).

Occurrence (MPEG Collection): Brazil: Pará (Parauapebas).

Mesocordylus papulatus (Fahraeus, 1845)

Material examined: 1 specimen. Brazil: Amazonas: Manaus, 1 km W Tarumã, 2-III-1981, C. Young Coll. (1).

Occurrence (MPEG Collection): Brazil: Amazonas (Manaus)

Mesocordylus scutellaris (Erichson, 1847)

Material examined: 2 specimens. Brazil: Acre: Porto Acre, Humaitá, Mata de Terra Firme, 15-VI to 2-VII-1992, I. S. Gorayeb \& J.A. Pena Colls (1). Pará: Benevides, 23-III-1993, J.A. Pena Coll. (1).

Occurrence (MPEG Collection): Brazil: Acre (Porto Acre); Pará (Benevides).

\section{Mesocordylus striatus (Boheman, 1838)}

Material examined: 8 specimens. Brazil: Maranhão: Lago Verde, 8-II-1983, R.B. Neto Coll. (1); Lago Verde, 2-III1983, W. França Coll. (1). Mato Grosso: Barra dos[do] Bugres, R. E. Serra das Araras, 23-24-I-1986, M. Zanuto Coll. (1). Pará: [without municipality], [?]-[?]-1900, Ducke Coll. (1); Belém, Utinga, 10-I-1978, M.F. Torres Coll. (1); Bragança, 15-I-1978, J. Batista Coll. (1); Peixe-Boi, 13-IV1977, M.F. Torres Coll. (1); Tucuruí, Rio Tocantins, margem direita, Chiqueirinho, 3-IV-1984, F.F. Ramos Coll. (1).

Occurrence (MPEG Collection): Brazil: Maranhão (Lago Verde); Mato Grosso (Barra do Bugres); Pará (Belém, Bragança, Peixe-Boi, Tucuruí).
Mesocordylus subulatus (Germar, 1824)

Material examined: 1 specimen. Brazil: Pará: Paragominas, Fazenda Cachoeira do Rio Vermelho, 15-I-1991, P. Tadeu Coll. (1).

Occurrence (MPEG Collection): Brazil: Pará (Paragominas).

\section{Orthognathus Schoenherr, 1838}

Orthognathus lividus Gyllenhal, 1838

Material examined: 8 specimens. Brazil: Goiás: Corumbá de Goiás, 3-I-1962, J. \& B. Bechyné Cols (1); same but, 4-II1962, (1). Mato Grosso: Chapada dos Guimarães, Fazenda Buriti, 17-II-1982, M. Zanuto \& W. Overal Colls (2), same but, 16-II-1982, W.L. Overal Coll. (1). Pará: Parauapebas, Serra Norte, N3, Armadilha Malayse, 9-12-II-1985 (1); Parauapebas, Serra Norte, Caldeirão, com luz, M.F. Torres Coll. (1); Pará: Parauapebas, Serra Norte, N1 (1).

Occurrence (MPEG Collection): Brazil: Goiás (Corumbá de Goiás); Mato Grosso (Chapada dos Guimarães); Pará (Parauapebas).

Orthognathus subparallelus (Chevrolat, 1880)

Material examined: 2 specimens. Brazil: Mato Grosso: Chapada dos Guimarães, Fazenda Buriti, 12-II-1982, M. Zanuto \& W. Overal Colls (1). Pará: Benevides, PA-408, km 06, flight trap, 1-V-1981, I.S. Gorayeb Coll. (1)

Occurrence (MPEG Collection): Brazil: Mato Grosso (Chapada dos Guimarães); Pará (Benevides).

\section{Rhinostomina Rhinostomus Rafinesque, 1815}

Rhinostomus barbirostris (Fabricius, 1775)

Material examined: 47 specimens. Brazil: Acre: Rio Branco, 25-X to 8-XI-1991, F.F. Ramos \& A. Henriques Colls (1). Amapá: [Laranjal do Jari], Cachoeira Aurucuotapá, Rio Jari, 18-VIII-1961, W. Egler Coll. (1); Laranjal do Jari, BR-156, km 14, Rio Matapi, 26-XI-1981, Coleta luz U.V., I.S. Gorayeb Coll. (4). Maranhão: Lago Verde, 7-XI-1983, W. França Coll. (1); Lago Verde, Lago Rodrigues, 25-V-1979, M.F. 
Torres Coll. (2). Mato Grosso: Serra dos [do] Bugres, Res. E. Serra das Araras, 24-I-1986, Armadilha Suspensa 1,6m (1). Pará: Belém, Ilha do Jutuba, 30-XI-1977, R. Ferreira Coll. (1); Belém, Campus MPEG, 3-II-1986, Sandoval Coll. (1); Belém, Campus MPEG, 11-III-1986, J. Dias Coll. (1); Belém, MPEG, 7-V-1974, B. Mascarenhas Coll. (1); Belém, Mocambo, 23-VII-1984, W.L. Overal Coll. (1); Belém, Utinga, 27-VI-1977, L. Hock Coll. (1); Bujaru, 21-IV-1979, R.B. Neto Coll. (1); Conceição do Araguaia, 17-21-XI-1979, R.B. Neto Coll. (4); Maracanã, Algodoal, 24-II-1990, J.M. da Rocha Coll. (1); Melgaço, Caxiuanã, ECFPn, $1^{\circ} 44$ " 27.6'/1 27" 34.2" W, 18-VIII-1995, R.M. Valente Coll. (1); Melgaço, Caxiuanã, ECFPn, 4-8-VIII-2000, H Ho \& C.K. Starr Colls (2); Óbidos, [?]-[?]-1906, P. Le Cointe Coll. (1); Parauapebas, Serra Norte, Igarapé Pojuca, 3-XI-1983, Coleta de Luz, F.F. Ramos Coll. (1); Parauapebas, Serra Norte, Manganês, 24-X-1984, Coleta Noturna, M.F. Torres Coll. (1); Primavera, Boa Vista, Ilha Arapiranga, Armadilha Malayse, 23-24-XI-1992, B. Mascarenhas Coll. (1); [Santarém], Taperinha, 24-I-1944 (1); Soure, 23-II-1979, S.A. Santos Coll. (1); Soure, 24-II-1979, P. Eremita Coll. (1); Soure, 24-II-197, S.A. Santos Coll. (1); Soure, 25-II-1979 (1); Soure, 25-II-1979, S.A. Santos Col (2); Soure, 25-II1979, P. Eremita Coll. (1); Soure, 25-II-1979, P. Nolasco Coll. (2); Soure, 27-II-1979, S.A. Santos Coll. (1); Tucuruí, Rio Tocantins, Ilha Tocantins, 21-VII-1984, B. Mascarenhas Coll. (2); Vigia, 11-VIII-1982, R.B. Neto Coll. (1). Rondônia: Ji-Paraná, 11-VIII-1984 (1); Ouro Preto do Oeste, Margem esquerda Rio Sta. Helena, 20-III-1985, M.F. Torres Coll. (1); Ouro Preto do Oeste, Reserva do INPA, 30-XI-1985, J. Dias Coll. (1); Ouro Preto do Oeste, Reserva do INPA, 24-VIII-1986, F.F. Ramos Coll. (1); Vilhena, 20-II-1961, J. \& B. Bechyné Colls (1). Without label (1).

Occurrence (MPEG Collection): Brazil: Acre (Rio Branco); Amapá (Laranjal do Jari); Maranhão (Lago Verde); Mato Grosso (Serra do Bugres); Pará (Belém, Bujaru, Conceição do Araguaia, Maracanã, Melgaço, Óbidos, Parauapebas, Primavera, Santarém, Soure, Tucuruí, Vigia); Rondônia (Ji-Paraná, Ouro Preto do Oeste, Vilhena).

\section{Rhynchophorini \\ Rhynchophorina Dynamis Chevrolat, 1883}

Dynamis borassi (Fabricius, 1801)

Material examined: 15 specimens. Brazil: Amazônia, without other information (1)*. Amazonas: Manaus, PDBFF / R-1302, [?]-[?]-1986, M. Martins Coll. (1); Manaus, 1 km W Tarumã 100 m, 16-II-1981, C. Young Coll. (1); [Santa Isabel do] Rio Negro, B. Cauaburi, 28-I-1963, J. \& B. Bechyné Colls (1). Pará: Acará, Ilha do Combu, 25-X-1989, R.B. Neto Coll. (1); same but, 26-X-1989 (1); Barcarena, Ilha das Onças, 30-I-1977, W.L. Overal Coll. (1); Belém, 19-IV-1980, M.F. Torres Coll. (1); Belém, Coqueiro, 3-VIII-1960, Inácio Coll. (1); Belém, P. MPEG, 6-III-1978, M.F. Torres Coll. (1); Belém, Mosqueiro, 16-VIII-1989, Coletado em palmeira, R.M. Valente Coll. (1); Belém [?], Pedreirinha, 8-IX-1960, Inácio Coll. (1)*; Breves, Ilha do Marajó, Rio Curuaçá, 6-III1988, J. Dias Coll. (1); Paragominas, Fazenda Cachoeira do Rio Vermelho, 16-I-1991, P. Tadeu Coll. (1); Santa Izabel[do Pará], 12-XII-1993, B. Mascarenhas Coll. (1).

Occurrence (MPEG Collection): Brazil: Amazonas (Manaus, Santa Isabel do Rio Negro); Pará(Acará, Barcarena, Belém, Breves, Paragominas, Santa Izabel do Pará).

\section{Dynamis nitidulus (Guérin, 1844)}

Material examined: 2 specimens. Brazil: Pará: Barcarena, Ilha das Onças, 7-II-1977, W.L. Overal Coll. (1); same but, 6-III-1977 (1).

Occurrence (MPEG Collection): Brazil: Pará (Barcarena).

\section{Dynamis peropacus Champion, 1910}

Material examined: 3 specimens. Brazil: Pará: Acará, $25-$ I-1978, P. Tadeu Coll. (1); Belém, Mocambo, 6-II-1979, F.F. Ramos Coll. (1); Belém, Mocambo, 31-I-1980, M.F. Torres Coll. (1).

Occurrence (MPEG Collection): Brazil: Pará (Acará, Belém). 


\section{Rhynchophorus Herbst, 1795}

Rhynchophorus palmarum (Linnaeus, 1758)

Material examined: 389 exemplares. Brazil: Acre: Alto Purus, [?]-[?]-1904, Ducke Col (1). Amapá: Itaubal do Piririm, Piririm, 19-X1975, Damasceno Coll. (1); Macapá, Base da FAB, 4-IV-1961, J. Flávio, C. Fr. Coll. (1); Mazagão, Camaipi km 14, 3-I-1980, E.L. Oliveira Coll. (1); [Oiapoque], [Vila Velha do] Cassiporé, [?]-[?]-1939 (3). Amazonas: Manaus, PDBFF, R-1302, [?]-[?]-1986, M. Martins Coll. (1); Maraã, Rio Japurá, Lago Paricá, 12-XI-1985, D.C. Neto Coll. (1); Maraã, Rio Japurá, Maguari, 11-X-1988, J. Dias Coll. (1); Presidente Figueiredo, UHH Balbina, 22-I-1988, B. Mascarenhas Coll. (1); [Santa Isabel do Rio Negro], Tapurucuara [Tapuruquara], Rio Negro, 2-II-1963, J. \& B. Bechyné Colls (1); same but, 4-II-1963 (4), 9-II-1963 (1), 24-IV-1964 (1). Maranhão: Buriticupu, 5-X-1978, R.B. Neto Coll. (1); Imperatriz, Ribeirãozinho, 9-VIII-1989, armadilha de luz, F.F. Ramos Coll. (1); Lago Verde, 10-III-1982, W.L. Overal Coll. (1); same but, 12-III-1982 (31); Lago Verde, 1-II-1983, R.B. Neto Coll. (10); same but, W. França Coll. (20); Lago Verde, 2-II-1983, R.B. Neto Coll. (8); same but, W. França Coll. (8); Lago Verde, 3-II-1983, R.B. Neto Coll. (2); same but, W. França Coll. (4); Lago Verde, 7-II-1983, W. França Coll. (1); Lago Verde, 8-II-1983, R.B. Neto Coll. (2); same but, W. França Coll. (1). Pará: Acará, Ilha do Combu, 11-VIII-1989, R.B. Neto Coll. (1); same but, 12-VIII-1989 (4), 15-VIII-1989 (7), 28VIII-1989 (7); Ananindeua, Fazenda da Pirelli, 18-IV-1990, R.B. Neto Coll. (2); Belém, 3-VII-1961, J. \& B. Bechyné, Colls (1); Belém, 28-VI-1962, Fer. C, Pinto Coll. (3); Belém, 8-VII-1962, A. Souza Coll. (1); Belém, 23-IV-1974, Fernanda Coll. (1); Belém, 18-IX-1976, Fernanda Coll. (1); Belém, 30-VII-1977, A.Y. Harada Coll. (1); Belém, 7-VIII-1977, M.F Torres Coll. (1); Belém, 10-IX-1977, F. Nascimento Coll. (1); Belém, 20-I-1978, M.F. Torres Col (1); Belém, 21-V-1979, M.F. Torres Col (1); Belém, Campus FCAP, 23-III-2001, L.A.S Sousa Col (4); Belém, Campus MPEG, 9-X-1990, M. Zanuto Coll. (1); Belém, Campus MPEG, 20-XII-1997, Moacir Ribeiro Coll. (1); Belém, Campus MPEG, 9-IX-2001 (1); same but, 12-IX-2001 (1); Belém, Cotijuba, 3-IX-1994,
J. Dias Coll. (1); Belém, EMBRAPA, 6-V-1978, H. Estevam (3); Belém, IAN, 29-VIII-1960, Inácio Coll. (1); same but, 26-X-1960 (1); Belém, Ilha Grande, 4-IX-1973, J. Queiroz Coll. (1); Belém, km 07, E. F. B., 8-X-1960, Inácio Coll. (2); Belém, Mocambo, 12-VI-1977, W.L. Overal Coll. (4); Belém, Mocambo, 6-VII-1977, P. Tadeu Coll. (1); same but, 6-VI-1978, (1); Belém, Mocambo, 13-VI-1978, Pe. Romeu Coll. (1); Belém, Mocambo, 2-V-1981, Luiz Gouvea Coll. (1); Belém, Mosqueiro, 16-VIII-1989, coletado em palmeira, R.M. Valente Coll. (1); Belém, Parque MPEG, 20-III-1978, M.F. Torres Coll. (1); Belém, Parque MPEG, 9-VII-1981, E. Melo Coll. (1); Belém, Parque MPEG, 23-VII-1985, M. Zanuto Coll. (1); Belém, Utinga, 1-IX-1960, Inácio (1); Belém, Utinga, 7-IX-1947 (1); same but, 8-IX-1947 (3), 9-IX-1947 (1); Belém, Utinga, [?]-III-1996, A. Souza Col (2); Benevides, Benfica, 11-V-1961, Bechyné Coll. (1); Breves, Marajó, Rio Curuaçá, B6, área 1, 7-Il-1988, J. Dias Coll. (1); Breves, Marajó, Rio Curuaçá, margem SE, 22-II-1988, isca 1, solo (2); Breves, Marajó, Rio Curuaçá, margem W, 22-II-1988, Isca 1, solo (1); Breves, Marajó, Rio Curuaçá, linha 1, 22-II1988, Isca: abacaxi (1); Breves, Marajó, Rio Curuaçá, área 2, 22-II-1988, Isca 1, coleta solo (1); Breves, Marajó, Rio Curuaçá, área 2, linha 2, 22-II-1988, Isca: abacaxi (1); Bujaru, 4-VI-1981, C.N. Arcanjo Coll. (1) (4); Bujaru, 3-VI-1982, W. França Coll. (2); Bujaru, 4-VI-1982 (1); Castanhal, 3-IV-1977, C.A. Batista Coll. (1); Conceição do Araguaia, 17-21-XI1979, R.B. Neto Coll. (6); same but, W. França Coll. (2), P. Maurício Coll. (1); Melgaço, Caxiuanã, 20-V-1991, Janduarir Coll. (1); Moju, Fazenda Latoniz, 25-V-1994, J. Dias Coll. (1); Ourilândia do Norte, Gorotire, Xingu, 14-XI-1977, D.A. Pasly Coll. (1); Parauapebas, Serra Norte, 3 alfa, 18-X-1986 (1); Parauapebas, Serra Norte, Manganês, 18-IX-1985, F.F. Ramos Coll. (1); Parauapebas, Serra Norte, Manganês, 13-IX-1986, armadilha Shanon, J. Dias Coll. (1); Peixe-Boi, 22-IV-1978, H. Estevam Coll. (3); Peixe-Boi, 24-IV-1978, W. França Coll. (1); Redenção, Ribeirão do Fogo, 20-XI, N.H. Rosa Coll. (1); São João de Pirabas, 26-VII-1947, M.F. Torres Coll. (1); same but, 25-VII-1978 (7), 26-VII-1978 (8), 27-VII-1978 (2); São João de Pirabas, Japerica, 22-XII-1992, 
J. Dias Coll. (1); São Miguel do Guamá, 24-III-1964, A. Souza Coll. (2); São Miguel do Guamá, 24-IV-1964, W. França Coll. (1); Tucuruí, 20-VI-1980, palmeira inajá, B. Mascarenhas Coll. (6); same but 17-VI-1981 (7); Tucuruí, Ilha, 8-VII-1984, B. Mascarenhas Coll. (1); same but, 21VII-1984 (1), 24-VII-1984 (4), 27-VII-1984 (5), 28-VII-1984 (1), 27-VIII-1984 (2); Tucuruí, llha, 27-VII-1984, W.L. Overal Coll. (4); Tucuruí, Margem esquerda, 13-III-1979, R.B. Neto Coll. (2); Tucuruí, Rio Tocantins, 3-III-1984, R.B. Neto Coll. (2); Tucuruí, Rio Tocantins, $70 \mathrm{~km}$ ao Sul de Tucuruí, 9-IV1984, M.F. Torres Coll. (5); Tucuruí, Rio Tocantins, Canoal, 28-III-1984, M.F. Torres Coll. (1), same but, 31-III-1984 (5); Tucuruí, Rio Tocantins, Chiqueirão, 6-IV-1984, M.F. Torres Coll. (2); same but, 7-IV-1984 (4), 8-IV-1984 (1), 9-IV-1984 (1), 11-IV-1984 (2); Tucuruí, Rio Tocantins, Chiqueirinho, 7-IV-1984, M.F. Torres Coll. (1), same but, 9-IV-1984 (1), 10-IV-1984 (4); Tucuruí, Rio Tocantins, Ilha Tocantins, 20VII-1984, B. Mascarenhas Coll. (2); same but, 21-VII-1984 (3), 26-VII-1984 (2), 28-VIII-1984 (1), 3-VIII-1984 (1); Tucuruí, Rio Tocantins, Jacundá, 7-V-1981, W. França Coll. (1); same but 29-IV-1984 (13), 2-V-1984 (1), 7-V-1984 (5), 8-V-1984 (1), 10-V-1984 (1), 13-V-1984 (2), 17-V-1984 (2); Tucuruí, Rio Tocantins, Jacundá, 1-V-1984, C.N. Arcanjo Coll. (1); same but, 2-V-1984 (2), 4-V-1984 (4), 5-V-1984 (2), 6-V-1984 (5), 8-V-1984 (1), 16-V-1984 (1), 17-V-1984 (4); Tucuruí, Rio Tocantins, Jacundá, 30-V-1984, W.L. Overal Coll. (3); Tucuruí, Rio Tocantins, Jacundá, Margem direita, 6-V-1984, C.N. Arcanjo Coll. (5); Tucuruí, Rio Tocantins, Margem direita; 10-III-1984, W.L. Overal Coll. (4); Tucuruí, Rio Tocantins, Saúde, 11-13-VI-1984, F. F. Ramos Coll. (1); [without municipality], 27-VI-1994 (1); [without municipality], [without date] (1); Tracuateua, Bragança, 4-XI-1990 (1); Tracuateua, Bragança, Santa Maria, 1-X-1988, I.S. Gorayeb Coll. (1). Pará/Amapá: Boca do Carecurú, Rio Jari, 9-VIII1961, W. Egler Coll. (1). Rondônia: [Candeias do Jamari], Rio Janari [Jamari], 5-11-XII-1988, N. Degalier Coll. (5); Ji-Paraná, 23-XI-1984, F.F. Ramos Coll. (1); Ouro Preto do Oeste, 14XI-1984, R.B. Neto Col (1); Ouro Preto do Oeste, Linha 212, 8-IV-1985, J. Dias Coll. (6); Ouro Preto do Oeste, Linha
62, km 16, 9-XI-1984, R.B. Neto Coll. (1); Ouro Preto do Oeste, Margem direita Rio Santa Helena, 21-III-1985, M.F. Torres Coll. (1). [Without label] (10)*.

Occurrence (MPEG Collection): Brazil: Acre (Alto Purus); Amapá (Itaubal do Piririm, Macapá, Mazagão, Oiapoque); Amazonas (Manaus, Maraã, Presidente Figueiredo, Santa Isabel do Rio Negro); Maranhão(Buriticupu, Imperatriz, Lago Verde); Pará (Acará, Ananindeua, Belém, Benevides, Breves, Bujaru, Castanhal, Conceição do Araguaia, Melgaço, Moju, Ourilândia do Norte, Parauapebas, Peixe-Boi, Redenção, São João de Pirabas, São Miguel do Guamá, Tucuruí, Tracuateua); Rondônia (Candeias do Jamari, Ji-Paraná, Ouro Preto do Oeste).

\section{Litosomina \\ Sitophilus Schoenherr, 1838}

Sitophilus zeamais Motschulsky, 1855

Material examined: 11 specimens. Brazil: Amapá: [Oiapoque], Serra Lombard [Serra Lombarda], Limão, 15VIII-1961, J. \& B. Bechyné Colls (1); same but 25-VIII-1961 (1); Goiás: Corumbá de Goiás, 5-II-1962, J. \& B. Bechyné Colls (1); Mato Grosso: Chapada dos Guimarães, 30-I1961, J. \& B. Bechyné Colls (1); same but, 5-II-1961 (1), 6-II-1961 (1); Cuiabá, 22-III-1961, J. \& B. Bechyné Colls (1); Pará: Belém, 8-I-1961, J. \& B. Bechyné Colls (1), 28-VI1962, J. \& B. Bechyné Colls (1); Benevides, 20-XI-1961, J. \& B. Bechyné Colls (1); Santa Izabel [do Pará], 23-VI-1962, J. \& B. Bechyné Colls (1).

Occurrence (MPEG Collection): Brazil: Amapá (Oiapoque); Goiás (Corumbá de Goías); Mato Grosso (Chapada dos Guimarães, Cuiabá); Pará (Belém, Benevides, Santa Izabel do Pará).

\section{Toxorhinus Lacordaire, 1866}

Toxorhinus banonii (Guérin, 1844)

Material examined: 1 specimen. Brazil: Pará: Tucuruí, Rio Tocantins, Saúde, armadilha suspensa 2 m, 1-3-VI-1984 (1). Occurrence (MPEG Collection): Brazil: Pará (Tucuruí). 


\section{Sphenophorina}

\section{Belopoeus Schoenherr, 1838}

Belopoeus carmelita Gyllenhal, 1838

Material examined: 10 specimens. Brazil: Pará: Melgaço, Caxiuanã, ECFPn, 18-VIII-1998, em inflorescência de Attalea maripa (Aubl.) Mart., R.M. Valente Coll. (5); same but, 18-VIII-1998 (5).

Occurrence (MPEG Collection): Brazil: Pará (Melgaço).

\section{Belopoeus caudatus Vanin, 1995}

Material examined: 2 specimens. Brazil: Pará: Melgaço, Caxiuanã, ECFPn, em inflorescência de Attalea maripa, 19-VIII-1998, R.M. Valente Coll. (2).

Occurrence (MPEG Collection): Brazil: Pará (Melgaço).

\section{Cactophagus Le Conte, 1876}

Cactophagus limulus (Vaurie, 1967)

Material examined: 2 specimens. Brazil: Pará: Belém, Utinga, 14-I-1954, W. França Coll. (1); Bujaru, Rodovia PA-140, km 26, 28-XII-1983, L.B. Albuquerque Coll. (1). Occurrence (MPEG Collection): Brazil: Pará (Belém, Bujaru).

\section{Cactophagus strigosus (Erichson, 1847)}

Material examined: 1 specimen. Brazil: Pará: Benevides, PA-408, km 06, 14-V-1981, E.L. Oliveira Coll. (1).

Occurrence (MPEG Collection): Brazil: Pará (Benevides).

\section{Cosmopolites Chevrolat, 1885}

\section{Cosmopolites sordidus (Germar, 1824)}

Material examined: 4 specimens. Brazil: Amapá: Mazagão, Camaipi km 14, 3-I-1980, E.L. Oliveira Coll. (1). Pará: Belém, 7-12-1960, Bechyné Coll. (1); Belém, 22-I-1963, F.C. Pinto Coll. (1); Belém, IAN, 26-X-1960, Inácio Coll. (1). Occurrence (MPEG Collection): Brazil: Amapá (Mazagão); Pará (Belém).

\section{Cyrtomasius Vanin, 1998}

Cyrtomasius marluciae Vanin, 1998 (Figure 1)

Vanin, S. A. (1998). Revista Brasileira de Entomologia 42(3-4): 111-120.

Material type examined: 11 paratypes. MPEG COL 15009587, male: "Brasil Pará/ C. grandiflora/Mocambo B11/ 03-IV-1987 [label 1], 0023 [label 2], Cyrtomasius/marluciae/ Paratype $\widehat{\sigma} /$ Vanin det. [label 3]". MPEG COL 15009588, male: "Brasil Pará/ Mocambo B38/1-II-1988 [label 1], Brasil Pará/ R. Santos [label 2], 0028 [label 3], C. grandiflora [label 4], Cyrtomasius/ marluciae/ Paratype $\widehat{\delta} /$ Vanin det. [label 5]". MPEG COL 15009589, male: "Brasil Pará/ Mocambo B38/28-I-1988 [label 1], Brasil Pará/R. Santos [label 2], 0029 [label 3], C. grandiflora [label 4], Cyrtomasius/ marluciae/ Paratype $\widehat{O} /$ Vanin det. [label 5]". MPEG COL 15009590, female: "Brasil Pará/ Mocambo/ B32/ 17-XI-1987 [label 1], Brasil Pará/M. Martins [label 2], 0021 [label 3], C. grandiflora [label 4], Cyrtomasius/marluciae/Paratype + /Vanin det. [label 5]". MPEG COL 15009591, female: "Brasil Pará/Mocambo/ B33/30-XI-1987 [label 1], Brasil Pará/ M. Martins [label 2], 0019 [label 3], C. grandiflora [label 4], Cyrtomasius/marluciae/ Paratype + / Vanin det. [label 5]". MPEG COL 15009810, male: "Brasil Pará Belém/ Mocambo/ B34/ 07-XII-1987 [label 1], Brasil Pará/M. Martins [label 2], C. grandiflora [label 3], Cyrtomasius/ marluciae/ Paratype $\hat{\jmath} /$ Vanin det. [label 4], MPEG [label 5, light blue]". MPEG COL 15009811, male: "Brasil Pará/ Mocambo/ B32/ 17-XI-1987 [label 1], Brasil Pará/M. Martins [label 2], C. grandiflora [label 3], Cyrtomasius/ marluciae/ Paratype $\delta /$ Vanin det. [label 4], MPEG [label 5, light blue]". MPEG COL 15009812, male; MPEG COL 15009813, male; MPEG COL 15009814, female, MPEG COL 15009815, female: "Brasil Pará/ Marajó-Breves/ Rio Caruacá/ 22-II-1988 [label 1], Américo/Clusia - sp. [label

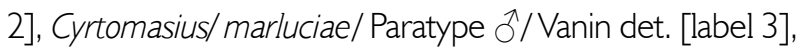
MPEG [label 4, light blue]".

Remarks: specimens pinned. Labels of identification rectangular, red, hand printed ink black. In the publication by Vanin (1998) is not mentioned that the paratype MPEG COL 15009588 is deposited at MPEG. In the Entomological 
Collection of the MPEG, all specimens received label with the follow: "Coleoptera: Polyphaga/ Curculionoidea: Curculionidae/ Rhynchophorinae/ Cyrtomasius marluciae/ Reincorporação: 4/XI/1998".

Additional material examined: 5 specimens. Brazil: Pará: Belém, Utinga, 15-Il-1965, H. Shubart Coll. (1); Belém, Utinga, 27-XII-1977, A.Y. Harada Coll. (1); Santa Bárbara, [?]-II-1965, A. Souza Coll. (3).

Occurrence (MPEG Collection): Brazil: Pará (Belém, Breves, Santa Bárbara).

\section{Foveolus Vaurie, 1968}

Foveolus anomalus Vaurie, 1968

Material examined: 9 specimens. Brazil: Pará: Tucuruí, 20-VI1980, Palmeira Inajá, B. Mascarenhas Coll. (1); Tucuruí, Rio Tocantins, Canoal, 13-III-1984, palmeira inajá, W.L. Overal Coll. (2); Tucuruí, Rio Tocantins, Canoal, 16-III-1984, em palmeira, W.L. Overal Coll. (1); Tucuruí, Rio Tocantins, margem esquerda, Canoal, 16-III-1984, W.L. Overal Coll. (3); Tucuruí, Rio Tocantins, Jacundá, 16-III-1984, W. França Coll. (1); Tucuruí, Rio Tocantins, Rio Arapari, 2-III-1984, W.L. Overal Coll. (1)

Occurrence (MPEG Collection): Brazil: Pará (Tucuruí).

\section{Foveolus maculatus O’Brien, 2003 (Figure 2)}

O'Brien, C. (2003). Transactions of the American Entomological Society 129(2): 329.

Material type examined: 3 paratypes, sex not determined. MPEG COL 15009601: "BRASIL-PARÁ/ EMBRAPA - A. oriental/Col.: R.N. MEDEIROS/3/IX/1996 [label 1], Foveolus maculatus/n. sp. O'Brien, 2003 [label 2], TRONCO DE AÇAí [label 3] 15003 [label 4] PARATYPE [label 5]". MPEG COL 15009602: same data. MPEG COL 15009603: "BRASIL-PARÁ/ EMBRAPA - CPATU/ A.A. SILVA / Data: 7/10/1997 [label 1], Açaí [label 2], 1503 [label 3], Foveolus maculatus/n. sp. O'Brien, 2003 [label 4], PARATYPE [label 5]".

Remarks: specimens pinned. Labels of paratype rectangular, red, printed. Labels of identification white, hand printed. In the publication by O'Brien (2003) is not mentioned that exactly these two paratypes are deposited at MPEG.

Occurrence (MPEG Collection): Brazil: Pará (Belém).

\section{Metamasius Horn, 1873}

\section{Metamasius anceps (Gyllenhal, 1838)}

Material examined: 1 specimen. Brazil: Acre: Porto Acre, Humaitá, Mata de Terra Firme, 15-VI to 2-VII-1992, I.S. Gorayeb \& J.A. Pena Colls (1).

Occurrence (MPEG Collection): Brazil: Acre (Porto Acre).

Metamasius cinnamominus (Perty, 1832)

Material examined: 68 specimens. Brazil: Amazonas: [Santa Isabel do] Rio Negro, B. Cauaburi, 28-I-1963, J. \& B. Bechyné Colls (1). Pará: Acará, Ilha do Combu, 15-VIII-1989, R.B. Neto Coll. (1), 16-VIII-1989 (7), 17-VIII-1989 (9); Acará, Ilha do Combu, 1-X-1989, Armadilha de Luz, R.B. Neto Coll. (3); Belém, 4-V-1991 (1); Belém, Campus MPEG, 23-II-1987, M. Zanuto Coll. (1); Belém, Fazenda Velha, 18-VIII-1970, T. Pimentel Coll. (1); Belém, IAN, 26-X-1960, Inácio Coll. (1); Belém, Mosqueiro, 22-VII-1989, R.M. Valente Coll. (1); Belém, Mosqueiro, 24-25-V-1997, M.L.J. Macambira Coll. (1); Bujaru, 21-I-1979, R. B. Neto Coll. (1), 20-IV-1979, (5) , 21-IV-1979 (9); Conceição do Araguaia, 17-21-XI-1979, W. França Coll. (2); same but, R.B. Neto Coll. (2); [without municipality], São José do Capim, 12-V-1977, F.S. Chagas (1)*; Tucuruí, 5-VI-1978, R.B. Neto Coll. (1); Tucuruí, 5-VII-1978 (1); same but, R.B. Neto Coll. (8), P. Tadeu Coll. (4); Tucuruí, 5-VIII-1978, R.B. Neto Coll. (3); Tucuruí, 7-V-1979 (1); Tucuruí, Rio Tocantins, Ilha Tocantins, 28-VII-1984, B. Mascarenhas Coll. (3).

Occurrence (MPEG Collection): Brazil: Amazonas (Santa Isabel do Rio Negro); Pará (Acará, Belém, Bujaru, Conceição do Araguaia, Tucuruí).

Metamasius crustosus Vaurie, 1966

Material examined: 8 specimens. Brazil: Maranhão: Lago Verde, 2-II-1983, W. França Col (1). Pará: [without

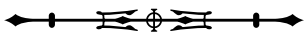


municipality, without date] (1)*; Belém, 1-VIII-1979, E. Melo Coll. (1); Belém, km 07, E.F.B., 21-X-1960, Inácio Coll. (1); Belém, Mosqueiro, 10-III-1990, C.N. Oliveira Coll. (1); Bujaru, 21-IV-1979, R.B. Neto Coll. (2); Conceição do Araguaia, 17-21-XI-1979, W.L. Overal Coll. (1).

Occurrence (MPEG Collection): Brazil: Maranhão (Lago Verde); Pará (Belém, Bujaru, Conceição do Araguaia).

\section{Metamasius hemipterus (Linnaeus, 1758)}

Material examined: 390 specimens. Brazil: Acre: Porto Acre, Humaitá, Mata de Terra Firme, 15-VI to 2-VII-1992, I.S. Gorayeb \& J.A. Pena Colls (1); Rio Branco, 25-X to8XI-1991, F.F. Ramos \& A. Henriques Colls. (1). Amapá: [Ferreira Gomes], Floresta IBDF [Floresta Nacional do Amapá], Rede, 19-II-1981, I.S. Gorayeb Coll. (1)*; Mazagão, Camaipi, km 14, 3-I-1980, E.D. Coll. (1); [Oiapoque], [Vila Velha do] Cassiporé, [?]-[?]-1939 (1). Amazonas: Itacoatiara, 30 km W, Rio Urubú, floresta primária, sweeping, day, 13-I1981, Robert Davidson Coll. (1); Itacoatiara, 30 km W, Rio Urubú, floresta primária, sweeping, day, 15-I-1981, Robert Davidson Coll. (1); [Santa Isabel do] Rio Negro, B. Cauaburi, 28-I-1963, J. \& B. Bechyné Colls (5). Goiás: Itaguari, [?]-IV1936 (1). Mato Grosso: Chapada dos Guimarães, 20-I-1961, J. \& B. Bechyné Colls (1). Pará: [without municipality] R. Ariranha, Trombetas, 8-10-193 [8-X-1903?], Ducke Coll. (1); Acará, Ilha do Combu, 11-VIII-1989, R.B. Neto Coll. (1); same but, 12-VIII-1989 (8), 15-VII-1989 (15), 16-VIII-1989 (12); Acará, Ilha do Combu, 15-VIII-1989, Armadilha de luz, R.B. Neto Coll. (1); same but, 1-X-1989 (1); Barcarena, 22I-1984, W. dos Santos Coll. (1); Barcarena, Ilha das Onças, 7-II-1977, W.L. Overal Coll. (1); Belém, 5-VII-1989, R.M. Valente Coll. (1); Belém, Campus MPEG, 2-V-1981, M. Zanuto Coll. (1); Belém, Campus MPEG, 12-VI-1989, J.A. Pena \& Deone Sá Colls (1); Belém, Campus MPEG, 20-VI1989, A. Henriques \& Deone Sá Colls (1); Belém, Campus MPEG, 12-VI-1993, Luzanira Costa \& Marcio Souza Colls (1); Belém, Fazenda Velha, 18-VIII-1970, T. Pimentel Coll. (1); Belém, Fazenda Velha, 14-V-1977, A.Y. Harada Coll. (2); Belém, Mocambo, 6-V-1977, C. Fonseca Coll. (4); same but, W.L. Overal Coll. (1), A.Y. Harada Coll. (1), P. Tadeu Coll. (1); Belém, Mocambo, 26-VI-1977, W.L. Overal Coll. (2); Belém, Mocambo, 10-IX-1977, A.Y. Harada Coll. (1); Belém, Mocambo, 11-IV-1978. M.F. Torres Coll. (1); Belém, Mocambo, 13-IV-1978, Pe. Romeu Coll. (2); same but, 13VI-1978 (1); Belém, Mocambo, 2-V-1981. Luis Gouvea Coll. (1); Belém, Mosqueiro, 25-III-1991, F.A. Ferreira Coll. (1); Belém, Mosqueiro, 27-VII-1991, coletado em palmeira, R.M. Valente Coll. (8); same but, 28-VII-1991, R.M. Valente Coll. (5); Belém, Mosqueiro, Margem esquerda da Rodovia 2,5 km, Rio Mirim-mirim, 9-IV-1985, M. Zanuto Coll. (1); Belém, UFPA, 13-VIII-1991, R.M. Valente Coll. (1); Belém, Utinga, [?]-VIII-1947 (1); Belém, Utinga, 7-V-1977, R. Ferreira Coll. (1); Belém, Utinga, 8-V-1977, W.L. Overal Coll. (5); same but, H. Stevam Coll. (1), A.Y. Harada (1); Benevides, PA-408, km 06, 7-V-1982, W. França Coll. (1); Benevides, Fazenda Morelândia, 5-III-1987, J. Dias Coll. (5); Breves, Marajó, Rio Tapixaua, 18-11-V-1987, N. Degalier Coll. (1); Bujaru, 1-VII-1977, P. Waldir \& L. Broack Colls (1); Bujaru, 3-VI-1982, W. França Coll. (4); Bujaru, 16-IV-1982, R.B. Neto Coll. (1); Conceição do Araguaia, 17-21-XI-1979, R.B. Neto Coll. (4); same but, W. França Coll. (2); Limoeiro do Ajuru, 17-XII1978, W.L. Overal Coll. (1); Melgaço, Caxiuanã, ECFPn, 24-VIII-1994, Bernadete Coll. (1), 17-VII-1995, R.M. Valente Coll. (1); Moju, Fazenda Latoniz, 25-V-1994, J. Dias Coll. (1); Ourilândia do Norte, Gorotire, Xingu, 14-XII-1977, D.A. Posey Col (1); Parauapebas, Serra Norte, Igarapé Pojuca, 3-XI-1983, I.S. Gorayeb Coll. (1), Parauapebas, Serra Norte, Serraria, 7-10-IX-1983 (1); Primavera, 18-II-1982, Palmeira Inajá, M. Zanuto Coll. (1); Óbidos, [?[-[?]-1906, P. Le Cointe Coll. (1); Tucuruí, 6-VII-1978, W.L. Overal Coll. (1); Tucuruí, 17-VI-1981, Palmeira Inajá, B. Mascarenhas Coll. (2); Tucuruí, Rio Tocantins, 3-III-1984, R.B. Neto Coll. (1); Tucuruí, Rio Tocantins, 1-VIII-1984, armadilha luz, B. Mascarenhas Coll. (1); Tucuruí, Rio Tocantins, Canoal, 31-I-1984, T. Pimentel Coll. (4); same but, B. Mascarenhas Coll. (1); Tucuruí, Rio Tocantins, Canoal, 16-III-1984, W.L. Overal Coll. (1); Tucuruí, Rio Tocantins, Canoal, 1-V-1984, W. França Coll. (1); Tucuruí, Rio Tocantins, Chiqueirão, 28-VII-1984, M.F. Torres Coll.

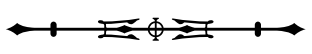


(1); Tucuruí, Rio Tocantins, Chiqueirinho, 7-IV-1984, M.F. Torres Coll. (4); Tucuruí, Rio Tocantins, Ilha, 24-III-1984, B. Mascarenhas (1); Tucuruí, Rio Tocantins, Ilha, 25-VII-1984, B. Mascarenhas (1); Tucuruí, Rio Tocantins, Ilha, 27-VII-1984, B. Mascarenhas (1); Tucuruí, Rio Tocantins, llha Tocantins, 19-VII-1984, B. Mascarenhas Coll. (1); Tucuruí, Rio Tocantins, Ilha Tocantins, 20-VII-1984, W. França Coll. (1); Tucuruí, Rio Tocantins, Ilha Tocantins, 24-VII-1984, B. Mascarenhas Coll. (1); Tucuruí, Rio Tocantins, Ilha Tocantins, 25-VII1984, B. Mascarenhas Coll. (1); Tucuruí, Rio Tocantins, Ilha Tocantins, 27-VII-1984, B. Mascarenhas Coll. (1); Tucuruí, Rio Tocantins, Ilha Tocantins, 28-VII-1984, B. Mascarenhas (3); Tucuruí, Rio Tocantins, Jacundá, 7-IV-1984, W. França Coll. (2); Tucuruí, Rio Tocantins, Jacundá, 28-IV-1984 (4); same but, W. França Coll. (47); Tucuruí, Rio Tocantins, Jacundá, 1-V-1984, W. França Coll. (24); Tucuruí, Rio Tocantins, Jacundá, 4-V-1984 (3); same but, W. França Coll. (2); Tucuruí, Rio Tocantins, Jacundá, 5-V-1984 (1); same but, W. França Coll. (14), C.N. Arcanjo Coll. (7); Tucuruí, Rio Tocantins, Jacundá, 6-V-1984, W. L. Overal Coll. (1); Tucuruí, Rio Tocantins, Jacundá, 7-V-1984 (9); same but W. França Coll. (9); Tucuruí, Rio Tocantins, Jacundá, 8-V-1984, W. França Coll. (8); Tucuruí, Rio Tocantins, Jacundá, 13-V1984, W. França Coll. (1); Tucuruí, Rio Tocantins, Jacundá, 15-V-1984, W. França Coll. (1); Tucuruí, Rio Tocantins, Jacundá, 16-V-1984, C.N. Arcanjo Coll. (5); same but, W. França Coll. (6); Tucuruí, Rio Tocantins, Jacundá, 17-V1984, W. França Coll. (18); same but, C.N. Arcanjo Coll. (2); Tucuruí, Rio Tocantins, Jacundá, 28-V-1984, W. França Coll. (4); Tucuruí, Rio Tocantins, Jacundá, 30-V-1984, W. França Coll. (3); same but W.L. Overal Coll. (4); Tucuruí, Rio Tocantins, Margem direita, 29-IV-1984 (3); same but, W.L. Overal Coll. (23), W. França Coll. (8); Tucuruí, Rio Tocantins, Margem direita, 4-V-1984, W. França (1); Tucuruí, Rio Tocantins, Margem direita, Jacundá, 1-V-1984, C.N. Arcanjo Coll. (8); Tucuruí, Rio Tocantins, Margem direita, 2 km ao Sul de Jacundá, 7-V-1984, C.N. Arcanjo Coll. (1); Tucuruí, Rio Tocantins, Rio Amapari, 27-IV-1984, T. Pimentel Coll. (2); Tucuruí, Rio Tocantins, Rio Arapari, 27-III-1984,
F.F. Ramos Coll. (2); Tucuruí, Rio Tocantins, Saúde, 9-11-VI1984, armadilha suspensa (1); Paraíba: João Pessoa, Reserva do Buraquinho IBDF, Armadilha Malayse, 25-29-I-1989, Y. Ada Coll. (1). Rondônia: Ouro Preto do Oeste, Reserva do INPA, 25-III-1985, W. França Coll. (1); Ouro Preto do Oeste, Margem direita, Rio Paraíso, 17-III-1985, M.F. Torres Coll. (2); Ouro Preto do Oeste, Margem direita, Sítio Deus é Amor, 26-III-1985, M.F. Torres Coll. (1); Ouro Preto do Oeste, Margem esquerda, Rio Sant Helena, 20-III-1985, M.F. Torres Coll. (1).

Occurrence (MPEG Collection): Brazil: Acre (Porto Acre, Rio Branco); Amapá (Ferreira Gomes, Mazagão, Oiapoque); Amazonas (Itacoatiara, Santa Isabel do Rio Negro); Goiás (Itaguari); Mato Grosso (Chapada dos Guimarães); Pará (Acará, Barcarena, Belém, Benevides, Breves, Bujaru, Conceição do Araguaia, Limoeiro do Ajuru, Melgaço, Moju, Ourilândia do Norte, Parauapebas, Primavera, Óbidos, Tucuruí); Paraíba (João Pessoa); Rondônia (Ouro Preto do Oeste).

\section{Metamasius tectus Vaurie, 1966}

Material examined: 10 specimens. Brazil: Acre: Porto Acre, Humaitá, Mata de Terra Firme, 15-VI to 2-VII- 1992, I.S. Gorayeb \& J.A. Pena Colls (1). Amazonas: São Gabriel da Cachoeira, Maturacá, Rio Negro, 9-I-1963, J. \& B. Bechyné Colls (1). Pará: Acará, llha do Combu, 16-VIII-1989, R.B. Neto Coll. (4), same but, 28-VIII-1989 (1); Belém, Utinga, 28-V-1977, A.Y. Harada Coll. (1); Tucuruí, Rio Tocantins, Ilha Tocantins, 28-VII-1984, B. Mascarenhas Coll. (1). Rondônia: Ouro Preto do Oeste, Estrada Rio Santa Helena, 23-III1985, M.F. Torres (1).

Occurrence (MPEG Collection): Brazil: Acre (Porto Acre); Amazonas (São Gabriel da Cachoeira); Pará (Acará, Belém, Tucuruí); Rondônia (Ouro Preto do Oeste).

\section{Metamasius tuberculipectus Hustache, 1936}

Material examined: 7 specimens. Brazil: Amazonas: Santo Antônio do Içá, 4-IX-1906, Ducke Coll. (1); São Gabriel da Cachoeira, Maturacá, Rio Negro, 6-I-1963, J. \& B. Bechyné

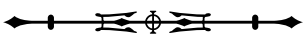


Colls (1); same but, 9-I-1963 (1), 12-I-1963 (1), 16-I-1963 (1). Pará: Almerim, Monte Dourado, 2-VI-1979, I.S. Gorayeb Coll. (1); Belém, Mocambo, 26-VI-1977, W.L. Overal Coll. (1).

Occurrence (MPEG Collection): Brazil: Amazonas (Santo Antônio do Içá, São Gabriel da Cachoeira); Pará (Almerim, Belém).

Metamasius spp.

Material examined: 7 specimens. Brazil: Amazonas: São Gabriel da Cachoeira, Maturacá, Rio Negro, 6-I-1963, J. \& B. Bechyné Colls (1); same but, 12-I-1963 (1). Pará: Acará, Ilha do Combu, 26-X-1989, R.B. Neto Coll. (1); Belém, Mocambo, 5-IV-1990, J. M. da Rocha Coll. (1); Benevides, 22-IV-1978, L. Texurie Coll. (1); Tucuruí, Rio Tocantins, 23-XII a 7-XII-1986, N. Degallier Col., Arm.[armadilha de] intercepção (2).

\section{Paramasius Kuschel, 1958}

Paramasius cristulatus Vanin, 1998 (Figure 3)

Material type examined: holotype, MPEG COL 15009592, male: "Brasil Pará/ Mocambo/ C55/20-XII-1990 [label 1], Brasil Pará/ F.F. Ramos [label 2], 0010 [label 3] Paramasius/ cristulatus/Holotype đ̊/Vanin det. [label 4]". 4 paratypes. MPEG COL 15009593, male: Brasil Pará/ Mocambo/ D06002/ 02-III-1991 [label 1], Brasil Pará/ F.F. Ramos [label 2], 0004 [label 3] Paramasius/cristulatus/ Paratype §/Vanin det. [label 4]". MPEG COL 15009594, female: "Brasil Pará Belém/ Mocambo/ E35015/ 26-V-1992 [label 1], Brasil Pará/ M. Martins [label 2], 0037 [label 3] Paramasius/ cristulatus/ Paratype $q /$ Vanin det. [label 4]". MPEG COL 15009595, female: "Brasil Pará/Marajó - Breves/ Rio Caruacá/ 22-II-1988 [label 1], Américo/ Clusia - sp. [label 2], 0011 [label 3] Paramasius/ cristulatus/ Paratype \&/Vanin det. [label 4]”. MPEG COL 15009596, male: "Brasil AM/Manaus PDBFF/R 1202 n8/M. Martins 1986 [label 1], 0012 [label 2], Paramasius/ cristulatus/ Paratype + /Vanin det. [label 3]".

Remarks: specimens pinned. Labels of identification rectangular, red, hand printed ink black. In the Entomological
Collection of the MPEG, all specimens received label with the follow: "Coleoptera: Polyphaga/ Curculionoidea: Curculionidae/ Rhynchophorinae/ Cyrtomasius marluciae/ Reincorporação: 4/XI/1998".

Additional material examined: 7 specimens. Brazil: Amapá: [Oiapoque], Serra Lombard [Serra Lombarda], Limão, 21-VIII-1961, J. \& B. Bechyné Coll. (1), same but, 24-VIII-1961 (1).

Occurrence (MPEG Collection): Brazil: Amazonas (Manaus); Amapá (Oiapoque); Pará (Belém, Breves).

Paramasius distortus (Gemminger \& Harold, 1871)

Material examined: 18 exemplares. Brazil: Amapá: Mazagão, Fazendinha, 9-XI-1978, W. França Coll. (1). Mato Grosso: Chapada dos Guimarães, Fazenda Buriti, 6-III-1982, M. Zanuto \& W.L. Overal Colls (1). Pará: Acará, llha do Combu, 12-VIII-1989, R.B. Neto Coll. (3); Altamira, 8-22-IV-1988, N. Degalier Coll. (1); Barcarena, Ilha das Onças, 6-III-1977, W.L. Overal Coll. (1); Belém, Fazenda Velha, 14-V-1977, M.F. Torres Coll. (1); Belém, Mocambo, 21-III-1978, M.F. Torres Coll. (1); Belém, Mocambo, C. grandiflora, 9-IX-1987 (1); Benevides, Fazenda Morelândia, 5-III-1987, J. Dias Coll. (1); same but, 6-III-1987 (3); Santa Bárbara, [?]-V-1964, W. França Coll. (2); São Miguel do Guamá, 24-III-1964, Apol. Souza Coll. (1); Belém-Brasília, km 90, F. Jaboti, 23-VII-1972, M. Helena Coll. (1)*.

Occurrence (MPEG Collection): Brazil: Amapá (Mazagão); Mato Grosso (Chapada dos Guimarães); Pará (Acará, Altamira, Barcarena, Belém, Benevides, Santa Bárbara, São Miguel do Guamá).

\section{Rhodobaenus Le Conte, 1876}

Rhodobaenus rufirostris (Hustache, 1936)

Material examined: 8 specimens. Brazil: Mato Grosso: Chapada dos Guimarães, 6-II-1961, J. \& B. Bechyné Colls (2). Pará: Parauapebas, Pojuca (2); Parauapebas, Caldeirão (1): Rondônia: Ouro Preto do Oeste, Margem esquerda Rio Sta. Helena, 20-III-1985, M.F. Torres Coll. (2); Ouro Preto do Oeste, Sítio Nova Lima, 22-III-1985, M.F. Torres Coll. (1). 
Occurrence (MPEG Collection): Brazil: Mato Grosso (Chapada dos Guimarães); Pará (Parauapebas); Rondônia (Ouro Preto do Oeste).

\section{Sphenophorus Schoenherr, 1838}

Sphenophorus sp. 1

Material examined: 1 specimen. Brazil: Pará: Acará, 8-XII1977, R.B. Neto Coll. (1).

\section{Sphenophorus sp. 2}

Material examined: 1 specimen. Brazil: Rondônia: Ji-Paraná, [FazendaSinueira]n84508, 27-VIII-1985, W. L. Overal Coll.(1).

\section{CONCLUSION}

The material examined in the Entomological Collection at Museu Paraense Emílio Goeldi represents a temporal and spatial record of Amazonian Dryophthorinae, including specimens collected in the early 1900s' and from regions already affected by human intervention. Additionally, this Collection includes the type specimens of three species of Dryophthorinae and the first recorded of the genus Rhodobaenus Le Conte, 1876 from Brazilian Amazon. Currently, $50 \%$ of the richness of the Dryophthorinae recorded from Brazilian Amazon is present in the Collection. This study highlights the importance of identifying and inventorying the material deposited in biological collections.

\section{ACKNOWLEDGMENTS}

We are thanks to Dr. Orlando Tobias Silveira, curator of the Entomological Collection of the Museu Paraense Emílio Goeldi, for granted complete access to the Collection; Augusto Quaresma and José Orlando Dias, for assistance in the Collection; MSc. Ronildon Miranda dos Santos for help with the map; MSc. Gustavo Costa Tavares for help with the illustrations; Dr. Bruno Augusto Souza de Medeiros for reviewing an earlier version of the manuscript and review English text. Our appreciation goes to the Museu Paraense Emílio Goeldi for providing facilities and access to the Entomological Collection.

\section{REFERENCES}

ALONSO-ZARAZAGA, M. A. \& C. H. C. LYAL, 1999. A world catalogue of families and genera of Curculionoidea (Insecta: Coleoptera) (excepting Scolytidae and Platypodidae): 5-315. Entomopraxis, Barcelona.

ALONSO-ZARAZAGA, M. A. \& C. H. C. LYAL, 2002a. Addenda and corrigenda to 'A world catalogue of families and genera of Curculionoidea (Insecta: Coleoptera)'. Zootaxa 63: 1-37. DOI: http:// dx.doi.org/10.11646/zoootaxa.63.1.1

ALONSO-ZARAZAGA, M. A. \& C. H. C. LYAL, 2002b. WTaxa: electronic catalogue of weevil names (Curculionoidea) (version 18, Dec 2017). Available at: http://wtaxa.csic.es/index.aspx. Accessed on: 2020, April 20.

ANDERSON, R. S., 2002a. Curculionidae. In: R. H. ARNETT JR., M. C. THOMAS, P. E. SKELLEY \& J. H. FRANK (Ed.): American beetles: Polyphaga: Scarabaeoidea through Curculionoidea: Vol. 2: 722-815. CRC Press, Boca Raton.

ANDERSON, R. S., 2002b. The Dryophthoridae of Costa Rica and Panama: checklist with new synonymy and descriptions of new species of Cactophagus, Mesocordylus, Metamasius and Rhodobaenus (Coleoptera; Curculionoidea). Zootaxa 80: 1-94. DOI: http://dx.doi. org/10.11646/zootaxa.80.1.1

ANDERSON, R. S., 2003. Neotropical Dryophthoridae: Redescription of the Genus Melchus Lacordaire with Description of Daisya Anderson, New Genus, and Seven New Species (Coleoptera: Curculionoidea) The Coleopterists Bulletin 57(4): 413-431. DOI: https://doi. org/10.1649/579

ANDERSON, R. S., 2008a. A review of the Neotropical genus Eucalandra Faust, 1899 (Coleoptera; Curculionidae: Dryophthorinae). Zootaxa 1791(1): 57-67. DOI: https://doi.org/10.11646/ zootaxa.1791.1.3

ANDERSON, R. S., 2008b. A review of the Neotropical genus Alloscolytroproctus Hustache, 1929 (Coleoptera; Curculionidae: Dryophthorinae). Zootaxa 1816(1): 35-43. DOI: https://doi. org/10.11646/zootaxa.1816.1.2

ANDERSON, R. S. \& A. E. MARVALDI, 2014. Dryophthorinae Schoenherr, 1825. In: R. A. B. LESCHEN \& R. G. BEUTEL (Ed.): Handbook of zoology, Arthropoda: Insecta: Coleoptera: morphology and systematics(Phytophaga): Vol. 3:477-483. Walter de Gruyter, Berlin.

BOUCHARD, P., Y. BOUSQUET, A. E. DAVIES, M. A. ALONSOZARAZAGA, J. F. LAWRENCE, C. H. C. LYAL, A. F. NEWTON, C. A. M. REID, M. SCHMITT, S. A. ŚLIPIŃSKI \& A. B. T. SMITH, 2011. Family-group names in Coleoptera (Insecta). Zookeys 88: 1-972. DOI: https://doi.org/10.3897/zookeys.88.807

CHAMORRO, M. L., 2019. An illustrated synoptic key and comparative morphology of the larvae of Dryophthorinae (Coleoptera, Curculionidae) genera with emphasis on the mouthparts. Diversity 11: 4. DOI: https://doi.org/10.3390/d11010004 
COSTA, C., S. IDE, G. H. ROSADO-NETO, M. H. M. GALILEO, C. R. V. FONSECA, R. M. VALENTE \& M. A. MONNÉ, 2000. Diagnóstico del conocimiento de las principales coleciones brasilenãs de Coleoptera. In: F. MARTÍN-PIERA, J. J. MORRONE \& A. MELIC (Ed.): Hacia un proyecto Cyted para el inventario y estimación de la diversidad entomológica en Iberoamérica: PrIBES-2000, Vol. 1: 115-136. Sociedad Entomológica Aragonesa SEA, Zaragoza.

INTERNATIONAL COMMISSION ON ZOOLOGICAL NOMENCLATURE (ICZN), 1999. International Code of Zoological Nomenclature. 4. ed. London, ICZN. Available at: www.nhm.ac.uk/ hosted-sites/iczn/code. Accessed on: 2019, December 5.

JORDAL, B. H., S. M. SMITH \&A. I. COGNATO, 2014. Classification of weevils as a data-driven science: leaving opinion behind. ZooKeys 439: 1-18. DOI: https://doi.org/10.3897/zookeys.439.8391

KUSCHEL, G., 1961. On problems of synonymy in the Sitophilus oryzae complex (30th contribution, Col. Curculionoidea). Annals and Magazine of Natural History 13(4): 241-244.

KUSCHEL, G., 1995. A phylogenetic classification of Curculionoidea to families and subfamilies. Memoirs of the Entomological Society of Washington 14: 5-33.

MARVALDI, A. E., 1997. Higher level phylogeny of Curculionidae (Coleoptera: Curculionoidea) based mainly on larval characters, with special reference to broad-nosed weevils. Cladistics 13: 285-312. DOI: https://doi.org/10.1111/j.1096-0031.1997.tb00321.x

MARVALDI, A. E. \& J. J. MORRONE, 2000. Phylogenetic systematics of weevils (Coleoptera: Curculionoidea): a reappraisal based on larval and adult morphology. Insect Systematics and Evolution 31: 43-58. DOI: https://doi.org/10.1163/187631200X00309

MARVALDI, A. E., A. S. SEQUEIRA, C. W. O'BRIEN \& B. D. FARRELL, 2002. Molecular and morphological phylogenetics of weevils (Coleoptera, Curculionoidea): do niche shifts accompany diversification? Systematic Biology 51: 761-785. DOI: https://doi. org/10.1080/10635150290102465

MCKENNA, D. D., A. S. SEQUEIRA, A. E. MARVALDI \& B. D. FARRELL, 2009. Temporal lags and overlap in the diversification of weevils and flowering plants. Proceedings of the National Academy of Sciences of the United States of America 106: 7083-7088. DOI: https://doi.org/10.1073/pnas.0810618106

MCKENNA, D. D., D. J. CLARKE, R. S. ANDERSON, J. J. ASTRIN, S. BROWN, M. L. CHAMORRO, S. R. DAVIS, B. DE MEDEIROS, M. G. DEL RIO, J. HARAN, G. KUSCHEL, N. M. FRANZ, B. JORDAL, A. LANTERI, R. A. B. LESCHEN, H. LETSCH, C. H. C. LYAL, A. E. MARVALDI, J. R. MERMUDES, R. G. OBERPRIELER, A. SCHÜTTE, A. SEQUEIRA, S. SHIN, M. H. VAN DAM \& G. ZHANG, 2018. Morphological and molecular perspectives on the phylogeny, evolution, and classification of weevils (Coleoptera: Curculionoidea): proceedings from the 2016 International Weevil Meeting. Diversity 10: 64. DOI: http://dx.doi.org/10.3390/d10030064
MORRONE, J. J. \& P. I. CUEVAS, 2009. On the status of the tribes Orthognathini and Rhinostomini (Coleoptera: Curculionidae: Dryophthorinae). Zootaxa 2216: 57-63. DOI: http://dx.doi. org/10.11646/zootaxa.2216.1.5

MUSEU PARAENSE EMÍLIO GOELDI (MPEG), [n. d.]. Available at: https://www.museu-goeldi.br/. Accessed on: 2019, December 5.

OBERPRIELER, R. G., A. E. MARVALDI \& R. S. ANDERSON, 2007. Weevils, weevils, weevils, everywhere. Zootaxa 1668: 491-520. DOI: http://dx.doi.org/10.11646/zootaxa.1668.1.24

OBERPRIElER, R. G., R. CALDARA, J. SKUHROVEC, A. E. MARVALDI, A. LANTERI, M. G. DEL RÍO, M. MEREGALLI, C. H. C. LYAL, H. A. HESPENHEIDE, N. M. FRANZ \& B. H. JORDAL, 2014. Curculionidae Latreille, 1802. In: R. A. B. LESCHEN \& R. G. BEUTEL (Ed.): Handbook of zoology, Arthropoda: Insecta: Coleoptera: morphology and systematics (Phytophaga): vol. 3: 423-648. Walter de Gruyter, Berlin.

O'BRIEN, C. W., 2003. Foveolus maculatus, a new species of palm weevil on Euterpe Mart. (Palmae) (Coleoptera, Dryophthoridae, Rhynchophorinae). Transactions of the American Entomological Society 129(2): 329-332.

ROSKOV, Y., G. OWER, T. ORRELL, D. NICOLSON, N. BAILLY, P. M. KIRK, T. BOURGOIN, R. E. DEWALT, W. DECOCK, E. VAN NIEUKERKEN \& L. PENEV, 2020. Species 2000 \& IT IS Catalogue of Life, 2020-02-24. Available at: www.catalogueoflife. org/col. Accessed on: 2020, April 20.

SHIN, S., D. J. CLARKE, A. R. LEMMON, E. M. LEMMON, A. L. AITKEN, S. HADDAD, B. D. FARRELL, A. E. MARVALDI, R. G. OBERPRIELER \& D. D. MCKENNA, 2018. Phylogenomic data yield new and robust insights into the phylogeny and evolution of weevils. Molecular Biology and Evolution 35: 823-836. DOI: https://doi.org/10.1093/molbev/msy057

SILVA, A. G. A., C. R. GONÇALVES, D. M. GALVÃO, A. J. L. GONÇALVES, J. GOMES, M. N. SILVA \& L. SIMONI, 1968. Quarto catálogo dos insetos que vivem nas plantas do Brasil. Seus parasitos e predadores: Parte 2, Tomo $^{\circ}{ }^{\circ}$, insetos, hospedeiros e inimigos naturais: 1-622. Ministério da Agricultura, Rio de Janeiro.

VALENTE, R. M., F. K. L. DIAMANTINO \& C. B. NUNES, 2005. The cholini (Curculionidae: Coleoptera) in the Museu Goeldi Collection. Boletim do Museu Paraense Emílio Goeldi. Série Ciências Naturais 1: 237-241.

VANIN, S. A., 1995. Three new species of palm weevils from the Amazonian region (Coleoptera, Curculionidae). Revista Brasileira de Entomologia 39: 871-881.

VANIN, S. A., 1998. A new genus and two new species of Sphenophorini from the Amazonian region, with notes on Paramasius distortus Gemminger \& Harold (Coleopetra, Curculionidae). Revista Brasileira de Entomologia 42: 111-120.

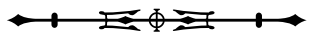


VANIN, S. A., D. C. BENÁ, H. L. RAINHO \& M. L. CHAMORRO, 2020. Catálogo Taxonômico da Fauna do Brasil. PNUD. Available at: http://fauna.jbrj.gov.br/fauna/faunadobrasil/2. Accessed on: 2020, April 20.

VAURIE, P., 1966. A revision of the Neotropical genus Metamasius (Coleoptera, Curculionidae, Rhynchophorinae). Species group I and II. Bulletin of the American Museum of Natural History 131: 211-337.

VAURIE, P., 1967a. A revision of the Neotropical genus Metamasius (Coleoptera, Curculionidae, Rhynchophorinae). Species group III. Bulletin of the American Museum of Natural History 136: 175-268.

VAURIE, P., 1967b. The Nawradii species group of Rhodobaenus (Coleoptera, Curculionidae, Rhynchophorinae). Species group III. American Museum Novitates 2310: 1-36.

VAURIE, P., 1968a. New weevils of the genus Metamasius form Central and South America (Coleoptera, Curculionidae, Rhynchophorinae). Species group III. American Museum Novitates 2316: 1-9.

VAURIE, P., 1968b. A new genus of weevils from South America (Coleoptera, Curculionidae, Rhynchophorinae. American Museum Novitates 2338: 1-14.

VAURIE, P., 1968c. Review of South American genus Belopoeus Schoenherr (Coleoptera, Curculionidae, Rhynchophorinae). The Coleopterists Bulletin 22: 39-44.
VAURIE, P., 1970a. Weevils of the tribe Sipalini (Coleoptera, Curculionidae, Rhynchophorinae). Part 1, The genera Rhinostomus and Yuccaborus. American Museum Novitates 2419: 1-57.

VAURIE, P., 1970b. Weevils of the tribe Sipalini (Coleoptera, Curculionidae, Rhynchophorinae). Part 2, The genera Mesocordylus and Orthognatus. American Museum Novitates 2441: 1-78.

VAURIE, P., 1971. Review of Scyphophorus (Curculionidae: Rhynchophorinae). The Coleopterists Bulletin 25(1): 1-8.

VAURIE, P., 1977. The Sipalini of the French Guiana (Coleoptera, Curculionidae, Rhynchphorinae). Bulletin du Muséum National d'Histoire Naturelle 412: 1227-1223.

VAURIE, P., 1978. Revision of the genus Sphenophorus in South America. American Museum Novitates 2656: 1-30.

VAURIE, P., 1980. A revision of Rhodobaenus. Part 1, Species in South America (Coleoptera, Curculionidae, Rhynchophorinae). Bulletin of the American Museum of Natural History 167: 1-44.

WATTANAPONGSIRI, A., 1966. A revision of the genera Rhynchophorus and Dynamis (Coleoptera, Curculionidae). Department of Agriculture Science Bulletin 1: 1-328.

WIBMER, G. J. \& C. W. O'BRIEN, 1986. Annotated checklist of the weevils (Curculionidae sensu lato) of South America (Coleoptera: Curculionoidea). Memoirs of the American Entomological Institute 39: 1-563.

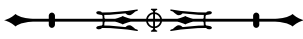

